\title{
Research on motion sensing interaction technology based on HTC Vive Fu-ping Deng ${ }^{1}$, Guang Hong ${ }^{2}$, Shuai Zhang ${ }^{3}$ and Bing Zhai ${ }^{4}$ \\ 1,2,3,4 Department 5, Wuhan Mechanical Engineering Academy, Wuhan, 430075 China
}

Keywords: motion sensing interaction, virtual reality, HTC Vive, quaternion.

\begin{abstract}
The motion sensing interactive devices can greatly enhance the sense of immersion. In this paper, the flow of the interaction with the HTC Vive is sums up five step. Analyzes the positioning principle of the HTC Lighthouse and introduce the function of the HTC thumbstick. Then defines the quaternion, and obtains the quaternion of changed state from HTC helmet and thumbstick in the real world. Calculates the visual angle and the picked model rotation in the virtual world according to the quaternion in the real world. Moreover designs multi person cooperative interaction model based on object model. Finally an interaction simulation system adopting HTC Vive device was achieved. The result shows the calculation method is correct.
\end{abstract}

\section{Introduction}

VR interactive devices can be the traditional type of devices (mouse, keyboard, handle, etc.) and motion sensing interaction devices. The interactive VR devices can greatly enhance the sense of immersion. Operator can make his body with the virtual world in a variety of interactive scene. Motion capture and spatial positioning technology are the core of motion sensing interactive, mainly including laser positioning technology, infrared optical positioning technology, optical positioning technology, computer vision, motion capture technology and inertial sensor based motion capture technology. The laser positioning technology has the advantage of low cost, high positioning accuracy, not blocked by occlusion, almost no delay, multiple target localization.HTC vive adopts the laser positioning technology, named Lighthouse [1].

\section{HTC vive interaction technology}

\section{The flow of the interaction}
A. Set pick scene and scene channel;
B. Get operator's position and direction.
C. Change the visual angle and visual position accordingly.
D. Get the picked object with HTC thumbstick and get the rotation of the thumbstick.
E. control the picked object' movement.

\section{HTC Lighthouse positioning principle}

HTC Lighthouse is a kind of laser scanning and positioning technology. It locates the track sensor in the operator by two laser sensors on the wall. Then it obtains the position and orientation information of the sensor.

Specifically, Lighthouse does not require the use of the camera, but by the laser and light sensitive sensors to locate the moving object. Two laser transmitters will be placed diagonally to form a rectangular area of $15 \times 15$ feet, which can be adjusted according to the actual size of the area. Laser beam emitted by the transmitter inside the two row of fixed LED lights, 6 times per second. Each laser is designed in a two scanning module, positioning space respectively in horizontal and vertical direction by scanning $15 \times 15$ feet in turns.

Vive HTC helmet and thumbstick have more than 70 light sensitive sensors. At the same time, the helmet starts counting, the sensor receives the laser, and the relationship between the position of the sensor and the time of receiving the laser is used to calculate the accurate position of the laser 
transmitter. If the laser beam hits the photosensitive sensor enough, then it will detect the position and the direction of the helmet.

\section{HTC Vive interaction principle}

HTC Vive interaction tool is a thumbstick[2] in figure 1.

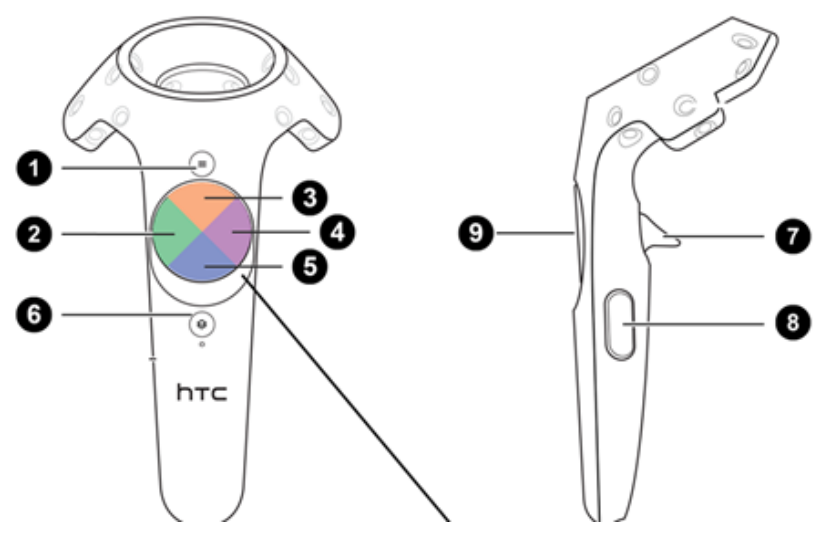

Figure 1 HTC thumbstick

1-Menu button (Shoulder), 2-Trackpad Left, 3-Trackpad Up, 4-Trackpad Right,

5-Trackpad Down, 6-System Button, 7-Trigger, 8-Grip Button, 9-Trackpad Press (Thumbstick)

In a simulation system, according to desire, program to control with HTC thumbstick instance and programmable button, make correlation between the object model's moveable state and HTC thumbstick's back coupling output. When operate HTC thumbstick, correlation controllable object model with synchronized motion. It has two operation models: 3D model style and visual point style.

According to the thinking of object-oriented, set up the object class that control HTC thumbstick, describe HTC thumbstick's attribute, respond to operation control. Adopt news response system to realize communicate between HTC thumbstick and system. In news handling function, judge the type of instance. Respond to every instance.

Mainly realize two kinds of control: transition control and rotation control. transition control is simple, which is one vector is added to the other vector.Howevre rotation control is complex, which is matrix operation wiht quaternion. If variable $\mathrm{q}$ is a quaternion[3],then

$q=\lambda+P_{1} i+P_{2} j+P_{3} k$

$i^{2}=j^{2}=k^{2}=i j k=-1$

A rigid body rotating the specific angle around some axis across a fixed point by point can reach any attitude.

if $n$ is the rotation axis and $\theta$ is the rotation angle then $\alpha, \beta, \gamma$,is the angel between projection of $n$ and coordinate aixs. The rotation axis is expressed as an identity vector

$\vec{n}=\cos \alpha \cdot i+\cos \beta \cdot j+\cos \gamma \cdot k=\cos \frac{\theta}{2}+\sin \frac{\theta}{2} \cos \alpha \cdot i+\sin \frac{\theta}{2} \cos \beta \cdot j+\sin \frac{\theta}{2} \cos \gamma \cdot k$

Then the rotation can be difined as a quaternion $\mathrm{q}$.

$$
q=\cos \frac{\theta}{2}+\sin \frac{\theta}{2} \cos \alpha \cdot i+\sin \frac{\theta}{2} \cos \beta \cdot j+\sin \frac{\theta}{2} \cos \gamma \cdot k
$$

When the operator with a helmet rotates his head in the real world ,it gives out two vector $R$ and $R^{\prime} . R$ is the operator's original direction and $R^{\prime}$ is the operator's final direction.Then the rotation $\mathrm{q}$ is described as

$$
R^{\prime}=q \cdot R \cdot q^{-1}
$$

The visual angle will rotate the same rotation in the virtual scene.

Homoplastically the wrist's rotation is the same to the helmet. When the operator rotates his wrist with a thumbstick in the real world, it gives out two vector $W R$ and $W R$ '. WR is the wrist's original direction and $W R$ ' is the wrist's final direction. Then the rotation q is described as 


$$
W R^{\prime}=q \cdot W R \cdot q^{-1}
$$

The picked model will rotate the same rotation in the virtual scene.

\section{Multi person cooperative interaction based on object model}

Many people wearing virtual reality device can gather together in the same scene, and the position of each person in the virtual scene are independent. Each can walk freely and view, also can be Cooperative operation.

Object model is composed of federation object model (FOM) and simulation object model (SOM). object model design is mainly object classes and interaction classes design essentially which include object class structure vobject class attributes interaction class structure and interaction class parameters, all the data upwards are embodied in FOM and SOM.

\section{Some object model design method}

A. Object model design method base on operation steps

The design idea of this method: we only design an "operation step object class" to every federate, it is published by local federate and subscribed by all other federates, it through object instance attributes update/reflection mechanism realize information interaction. After federate complete certain a step operation in federation execution, it update object instance attributes according to this step operation information through development engine. In the course of time advancement, federate reflect attribute values and synchronously update operation step information that be determinate by the reflected attribute values.

B. Object model design method base on operation parts object

The design idea of this method: according to the operation parts which participate in interaction among federates, we call it "operation parts object class", the operation parts object participate in interaction among federates in fact system decide the number of object classes, which is one to one corresponding relation in principle. According to the interaction relation among federates, federate decide what object classes need be published/subscribed. If there are published cross relation, federate will realize attributes update in federation execution through transfer mechanism of attribute ownership. The interaction information among federates is the state of operation component that certain a step operation come down to, accept side federate update scene use component states which have already been accept.

\section{Object model design method base on operation step and unilateralism interaction between}

\section{federates}

This method combines the virtue of object model design method base on operation step and operation parts object. It use the content of operation step to token the interaction information between federates. It adopt the method that unilateralism satisfy the interaction need of "acquire to supply". It eliminates meaningless information transfer by the mechanism of exclusive publisher and subscriber of class and reduces the amount of object classes and interaction classes in object models to the best of its ability.

\section{A. Design idea of this method}

For the specialty of operation training system, we treat operation step as main information among federates. There is a "acquire to supply" type unilateralism interaction exist between local federate and arbitrary other federate, this kind interaction relation present reticulation structure between three federates.

Transfer aspect must be from offer aspect to receive aspect; interaction information is only defined by one format that is operation steps information. To every unilateralism interaction, in the offer aspect's SOM, we design the only object class or interaction class corresponding to it, identifier of class should obviously express the unilateralism interaction relation between offer aspect and receive aspect. Object class attributes or interaction parameters lie on operation step information that be delivered between federates and material simulation need. In federation execution, offer aspect is the 
only publisher of object class or interaction class, according to simulation need, it only answer for update its object instance attribute. The receive aspect is the only effect subscriber of object class, it update events or interaction instances and parameters through development engine receive the object instance attributes what offer aspect sent. All federates create their SOM according to above method, FOM of federation can be acquired by combing all SOM of every federate.

B. FOM design

We treat operation step information as the main content of object class design when design SOM of every federate, furthermore, to the unilateralism interaction exist in arbitrary two federates, we should respectively design corresponding object classes to devise description, every object class has the same data structure. Combine the object classes of SOM of every federate can we acquire the object classes of FOM.

C. Class attributes design

Because all operation of federate have corresponding only operation step coding in operation rule overall form, so local federate only need send the corresponding operation step coding and influence relation type of other federates. So object class has two attributes: operation step number and influence relation type.

\section{Summary}

An interaction simulation system adopting HTC Vive device was achieved. The VR engine is Unity3D, it provides many functions for quaternion operation. One scene is shown in figure 2 . The result shows the calculation method for visula angle and picked model's moving track is correct.And the multi person cooperative interaction based on object model is effective.

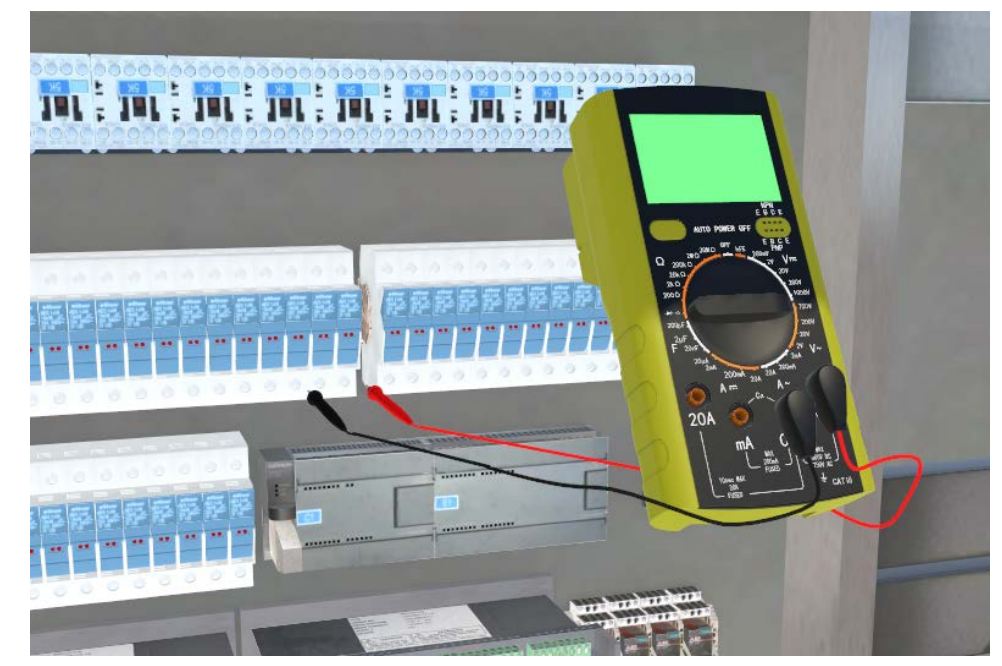

Figure 2 the effect figure

\section{References}

[1] Information on http://www.lighthouse-tech.com/

[2] Information on http://www.manew.com/thread-90681-1-1.html

[3] Information on https://en.wikipedia.org/wiki/Quaternion

[4] Wang J, Zhai S, Canny J. Camera phone based motion sensing: interaction techniques, applications and performance study[C].Proceedings of the 19th annual ACM symposium on User interface software and technology. ACM, 2006:101-110.

[5] Liang X, Zhang S, Li Q, et al. 3D Intuitive Gesture Interaction via Motion Sensing [J]. Journal of Computer-Aided Design \& Computer Graphics, 2010, 22(3):521-520. 